\title{
Noninvasive Serum Fibrosis Markers are Associated with Coronary Artery Calcification in Patients with Nonalcoholic Fatty Liver Disease
}

\author{
Do Seon Song ${ }^{1}$, U Im Chang ${ }^{1}$, Sung-Goo Kang ${ }^{2}$, Sang-Wook Song ${ }^{2}$, and Jin Mo Yang ${ }^{1}$ \\ Departments of ${ }^{1}$ Internal Medicine and ${ }^{2}$ Family Medicine, St. Vincent's Hospital, College of Medicine, The Catholic University of Korea, Suwon, \\ Korea
}

Background/Aims: Advanced hepatic fibrosis is associated with cardiovascular disease (CVD) in patients with nonalcoholic fatty liver disease (NAFLD). We investigated the association between noninvasive serum fibrosis markers and the coronary artery calcium score (CACS) in subjects with NAFLD. Methods: We analyzed 665 NAFLD subjects without chronic liver disease or heart disease between 2011 and 2015. The noninvasive fibrosis markers that were used to evaluate the severity of hepatic fibrosis included the NAFLD fibrosis score (NFS), fibrosis-4 (FIB-4) score, Forn's index, and the aspartate aminotransferase to platelet ratio index (APRI). Results: The areas under the receiver operating characteristics curves for the NFS, FIB-4 score, Forn's index and APRI for predicting CACS >100 were 0.689, 0.683, 0.659, and 0.595, respectively. According to the multivariate analysis, older age, increased body mass index (BMI), and decreased estimated glomerular filtration rate (eGFR) were significant factors associated with CACS $>100$. The NFS, FIB-4 score and APRI were significantly associated with CACS $>100$ after adjusting for age and gender $(p=0.006, p=0.012$, and $p=0.012$, respectively) and after adjusting for age, gender, BMI and eGFR $(p=0.013, p=0.022$, and $p=0.027$, respectively). Scores integrating noninvasive fibrosis markers and other risk factors improved the predictive accuracy. Conclusions: The NFS and FIB-4 score were associated with coronary atherosclerosis in subjects with NAFLD. Furthermore, scores integrating these noninvasive scores and risk factors for CVD showed good discriminatory power in predicting CACS $>100$. Therefore, noninvasive serum fibrosis markers may be useful tools for identifying NAFLD subjects at a high risk for CVD. (Gut Liver 2019;13:658-668)

Key Words: Non-alcoholic fatty liver disease; Coronary artery calcium score; Liver fibrosis marker

\section{INTRODUCTION}

Nonalcoholic fatty liver disease (NAFLD) is the most common cause of chronic liver disease worldwide, and is estimated to be the cause of approximately 25\% of chronic liver disease cases. ${ }^{1,2}$ NAFLD comprises a spectrum of pathological conditions, which range from simple steatosis to nonalcoholic steatohepatitis (NASH) and cirrhosis. NAFLD, especially NASH, can progress to advanced liver disease, leading to cirrhosis, liver failure, and hepatocellular carcinoma ${ }^{3-5}$ and is now a major cause of liverrelated morbidity and mortality. ${ }^{6}$

Although NAFLD comprises histological changes in the liver, its clinical burden is not confined to liver-related morbidity and mortality. NAFLD is now regarded as a multisystem disease associated with extrahepatic chronic diseases, such as diabetes, cardiovascular disease (CVD), and metabolic syndrome. Previous studies have reported that NAFLD is associated with subclinical atherosclerosis, such as coronary artery calcification ${ }^{7,8}$ and carotid intima-media thickness (CIMT). ${ }^{9}$ Moreover, in the natural course of NAFLD, the most important cause of death is CVD.,10 In previous studies, mortality from CVD in NAFLD patients has been shown to be associated with advanced fibrosis. ${ }^{3}$ Thus, identifying patients with advanced fibrosis among subjects with NAFLD is important in identifying those with a high risk of developing CVD.

Liver biopsy is considered a reference method for assessing the severity of inflammation and degree of fibrosis in NAFLD patients. However, liver biopsy is an invasive procedure that is associated with several complications, and the sampling error and inter- and intra-observer variability can be major issues. Because of these limitations, noninvasive methods to assess the

\footnotetext{
Correspondence to: Jin Mo Yang

Department of Internal Medicine, St. Vincent's Hospital, College of Medicine, The Catholic University of Korea, 93 Jungbu-daero, Paldal-gu, Suwon 16247, Korea

Tel: +82-31-249-8211, Fax: +82-31-253-8898, E-mail: jmyangdr@catholic.ac.kr Received on September 28, 2018. Revised on December 10, 2018. Accepted on December 24, 2018. Published online June $28,2019$. pISSN 1976-2283 eISSN 2005-1212 https://doi.org/10.5009/gnl18439

() This is an Open Access article distributed under the terms of the Creative Commons Attribution Non-Commercial License (http://creativecommons.org/licenses/by-nc/4.0) which permits unrestricted non-commercial use, distribution, and reproduction in any medium, provided the original work is properly cited.
} 
severity of hepatic fibrosis were developed using serum markers. Many serum biomarkers were developed and include combinations of direct markers that reflect extracellular matrix turnover and indirect markers that can be detected by simple biochemical tests. ${ }^{11-13}$ These biomarkers were validated in subjects with NAFLD in many studies and are now included in clinical practice guidelines to define the presence of advanced fibrosis. ${ }^{12,13}$

Therefore, in this cross-sectional study, we aimed to assess the association between noninvasive serum fibrosis markers and the coronary artery calcium score (CACS), which is a strong predictor of cardiovascular events, in subjects with NAFLD, as detected by ultrasonography.

\section{MATERIALS AND METHODS}

\section{Study population}

We conducted a retrospective, cross-sectional study on 34,890 asymptomatic subjects who received an abdominal ultrasound (US) as a health check-up at the Health Promotion Center of St. Vincent's Hospital, The Catholic University of Korea, Suwon, Korea, between January 2011 and December 2015. Subjects without evidence of fatty liver on US $(n=18,471)$ and those who did not undergo a coronary computed tomography (CT) scan $(n=15,501)$ were excluded. Coronary CT scan was performed on the subjects who wanted the test. Only data from the first checkup were included for subjects who underwent a health checkup more than twice $(n=48)$. We excluded subjects who met any of the following criteria: (1) average alcohol consumption $\geq 30$ $\mathrm{g} /$ day in men and $\geq 20 \mathrm{~g} /$ day in women ( $\mathrm{n}=126$ ); (2) hepatitis B surface antigen positive or undetermined hepatitis B surface antigen ( $\mathrm{n}=17)$; (3) hepatitis C antibody positive ( $\mathrm{n}=8$ ); (4) lack of laboratory data $(\mathrm{n}=28)$; and (5) previous history of heart disease ( $n=26)$. Finally, we analyzed 665 subjects in this study (Fig. 1).

The study was approved by the Institutional Review Board of St. Vincent's Hospital (VC17RESI0234).

\section{Abdominal US}

Abdominal US was performed using an ACUSON Sequoia 512 (Siemens Medical Solution, Mountain View, CA, USA) or EPIQ 5 (Philips Ultrasound, Bothell, WA, USA) by experienced radiologists at the Health Promotion Center of St. Vincent's Hospital. US examiners were blinded to the subjects' health status. Hepatic steatosis was diagnosed by the following criteria: (1) parenchymal brightness; (2) liver to kidney contrast; (3) deep beam attenuation; and (4) vessel blurring.

\section{Coronary CT imaging}

Coronary CT imaging was conducted using a 128-multislice scanner (Optima CT660; GE Healthcare Japan Corp., Tokyo, Japan) or dual source CT system (SOMATOM Definition Flash; Siemens Healthcare, Forchheim, Germany) with administration of 90 to $100 \mathrm{~mL}$ of iodinated contrast medium (Ultravist ${ }^{\circledR} 370$; Bayer Healthcare, Berlin, Germany). Images were reconstructed at a 3-mm slice thickness. CACS was calculated as described by Agatston et al. ${ }^{14}$ using the AW server 4.6 (GE Healthcare). In brief, calcium deposits with an attenuation of more than 130

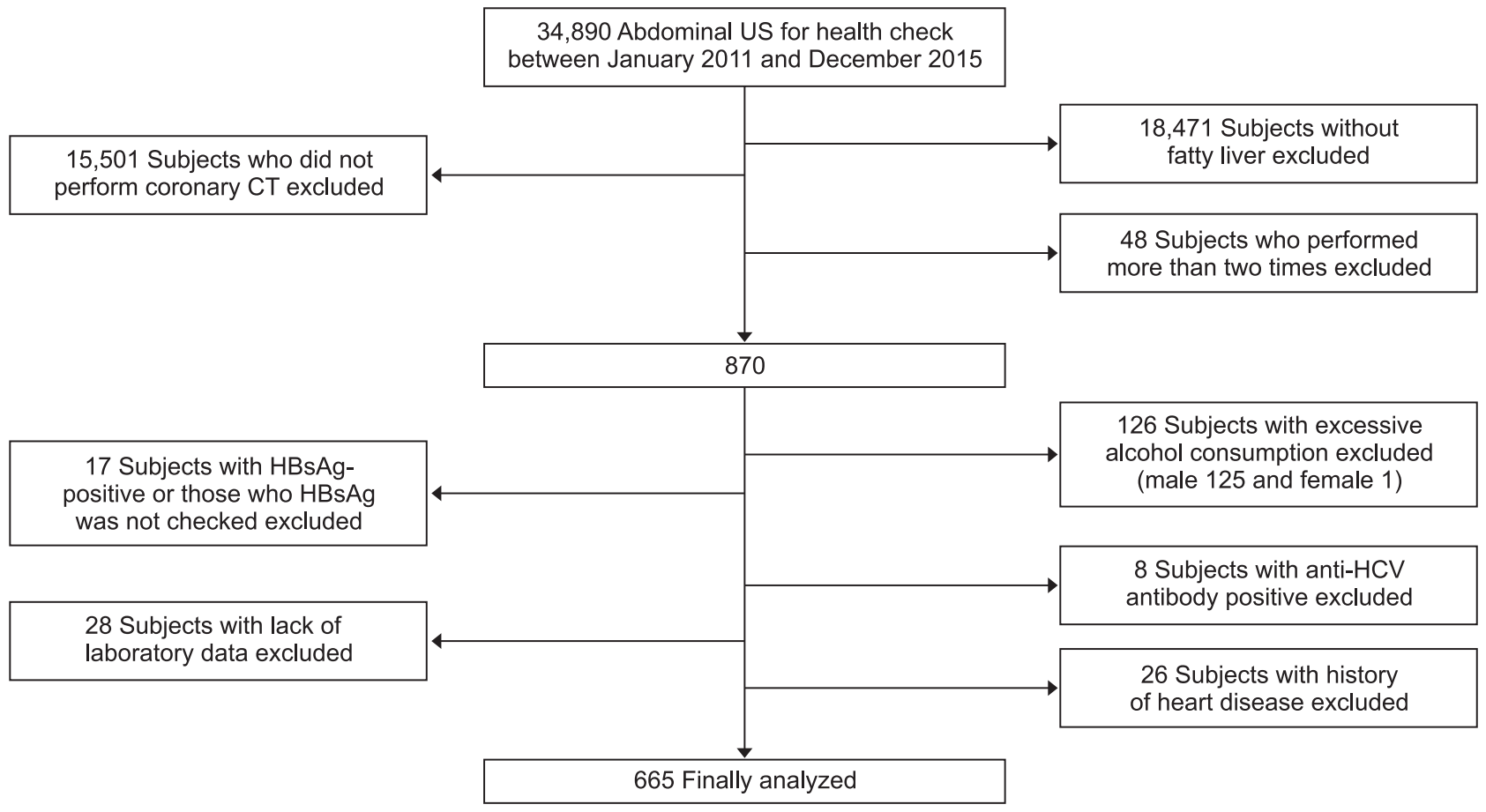

Fig. 1. Flow diagram of the determination of the study population. US, ultrasound; CT, computed tomography; HBsAg, hepatitis B surface antigen; HCV, hepatitis C virus. 
Hounsfield units (HU) are multiplied by a density weighting factor derived from the maximal $\mathrm{CT}$ attenuation within a given calcified lesion. The score for all lesions in all coronary arteries is then summed.

\section{Clinical and laboratory data}

The participants' demographic data and medical history, such as hypertension, diabetes, CVD, alcohol consumption, smoking status, and medication use, were obtained through a self-administered health questionnaire. The cutoff value of increased age was 55, which is a coronary artery disease (CAD) risk factor in women. ${ }^{15}$ Smoking status was categorized into two groups: current smokers and nonsmokers or past-smokers. Current smokers were defined as those who smoked at least one cigarette per day during the past 1 year. The participants were asked about their average frequency and amount of alcohol consumption for 1 year. The average amount of alcohol consumption per day was calculated, and men who consumed more than $30 \mathrm{~g} /$ day and women who consumed more than $20 \mathrm{~g} /$ day were excluded. Anthropometric measurements of the subjects were performed by trained nurses. Body weight and height were measured using a digital scale, and body mass index (BMI) was calculated as weight in kilograms divided by height in meters squared. The waist circumference was measured at the level of the midpoint between the iliac crest and costal margin. Subjects with a systolic blood pressure $\geq 140 \mathrm{~mm} \mathrm{Hg}$ or diastolic blood pressure $\geq 90 \mathrm{~mm} \mathrm{Hg}$ or history of antihypertensive drug use were defined as having hypertension. Subjects with a fasting serum glucose level $\geq 126 \mathrm{mg} / \mathrm{dL}$ or history of hypoglycemic agent use were defined as having diabetes mellitus. Laboratory data included albumin, bilirubin, aspartate aminotransferase (AST), alanine aminotransferase (ALT), gamma-glutamyl transpeptidase (GGT), total cholesterol, triglyceride, high-density lipoprotein, low-density lipoprotein, glucose, creatinine, platelet count, hepatitis B surface antigen, and antibody to hepatitis $C$ virus.

The estimated glomerular filtration rate (eGFR) was calculated by the Modification of Diet in Renal Disease equation as follows: $175 \times$ serum creatinine $(\mathrm{mg} / \mathrm{dL})^{-1.154} \times$ age (year) ${ }^{-0.203} \times 0.723$ (if female) $\times 1.212$ (if African American). ${ }^{16}$

\section{Noninvasive steatosis and fibrosis markers}

To assess the association between hepatic steatosis or fibrosis and CACS, we calculated the hepatic steatosis index $(\mathrm{HSI})^{17}$ and fatty liver index (FLI) ${ }^{18}$ as indexes of hepatic steatosis and NAFLD fibrosis score (NFS), ${ }^{19}$ Fibrosis-4 (FIB-4) score, ${ }^{20}$ Forn's index, ${ }^{21}$ and AST to platelet ratio index (APRI) ${ }^{22}$ as indexes of liver fibrosis. The HSI was calculated as $8 \times \mathrm{ALT} /$ AST+BMI (+ 2 if type 2 diabetes, +2 if female) and FLI was calculated as $\mathrm{e}^{\mathrm{y}} /\left(1+\mathrm{e}^{\mathrm{y}}\right) \times 100$, where $\mathrm{y}=0.953 \times \ln$ (triglycerides, $\mathrm{mg} /$ $\mathrm{dL})+0.139 \times\left(\mathrm{BMI}, \mathrm{kg} / \mathrm{m}^{2}\right)+0.718 \times \ln (\mathrm{GGT}, \mathrm{U} / \mathrm{L})+0.053 \times$ (waist circumference, $\mathrm{cm})-15.745$. The NFS was calculated as $-1.675+0.037 \times\left(\right.$ age, year) $+0.094 \times\left(\mathrm{BMI}, \mathrm{kg} / \mathrm{m}^{2}\right)+1.13 \times$ impaired fasting glucose/diabetes (yes=1, no=0)+0.99 $\times$ AST/ALT ratio$0.013 \times$ (platelet count, $\times 10^{9} / \mathrm{L}$ )- $0.66 \times$ (albumin, g/dL). The FIB-4 score was calculated as (agexAST)/(platelet count $\times$ [square root of ALT]). Forn's index was calculated by applying the following regression equation: $7.811-3.131 \times \ln$ (platelet count, $10^{9} / \mathrm{L}$ ) + $0.781 \times \ln ($ GGT, IU/L) + 3.467× $\ln ($ age, year) $-0.014 \times$ (cholesterol, $\mathrm{mg} / \mathrm{dL}$ ). The APRI was calculated based on the following formula: AST (IU/L)/AST upper limit of normal (IU/L)/platelet count in $\left(10^{9} / \mathrm{L}\right) \times 100$.

\section{Statistical analysis}

The data were expressed according to the properties of the variables. Continuous variables are presented as the means and standard deviations. Categorical variables are presented as frequencies and percentages. All subjects were categorized into three groups: $\mathrm{CACS}=0,>0-100$, and over 100. Categorical variables were compared using the chi-square test or Fisher exact test, and continuous variables were compared using oneway analysis of variance with Scheffe post hoc test. The association between clinical characteristics and increased CACS was evaluated using multinomial logistic regression analysis. In addition, to identify the risk factors for CACS $>100$, univariate and multivariate binary logistic regression analyses were performed using the backward elimination method (likelihood ratio). In multivariate analysis, statistically significant factors $(\mathrm{p}<0.05)$ and factors with near-marginal significance $(\mathrm{p}<0.10)$ were included. Because the noninvasive fibrosis scores included age, which is a significant risk factor, multi-collinearity could be present. Therefore, we performed multivariate analysis while excluding these scores. The accuracy in predicting CACS $>100$ was assessed by the area under the receiver operating characteristics (AUROC) curve. The AUROC curve was performed to calculate the sensitivity, specificity, positive predictive value (PPV), and negative predictive value (NPV). The cutoff point that maximized the Youden's index ( $\mathrm{J}=$ sensitivity+specificity-1) was chosen. Noninvasive steatosis and fibrosis scores were adjusted by age and gender (model 1) and adjusted by significant risk factors by multivariate analysis (model 2) using binary logistic regression analysis. A p-value less than 0.05 was considered to be statistically significant. Statistical analysis was performed using SPSS version 21.0 (IBM Corp., Armonk, NY, USA).

\section{RESULTS}

\section{Baseline characteristics}

The baseline characteristics of the participants are shown in Table 1. The mean age of all subjects was $51.5 \pm 9.3$ years, and 486 subjects (73.5\%) were male. The prevalence rates of hypertension, diabetes, and metabolic syndrome were 43.5\%, 14.1\%, and 51.7\%, respectively. Most subjects ( $n=485,72.9 \%)$ had no calcification in their coronary arteries (CACS=0), whereas 128 subjects (19.2\%) had a CACS between 0 and 100, and 52 sub- 
Table 1. Baseline Characteristics

\begin{tabular}{|c|c|c|c|c|c|}
\hline \multirow{2}{*}{ Characteristic } & \multirow{2}{*}{ Total $(n=665)$} & \multicolumn{3}{|c|}{ Coronary artery calcium score } & \multirow{2}{*}{ p-value } \\
\hline & & $0(\mathrm{n}=485)$ & $>0-100(n=128)$ & $>100(n=52)$ & \\
\hline Age, yr & $51.5 \pm 9.3$ & $49.7 \pm 8.7$ & $55.2 \pm 9.5^{*}$ & $58.7 \pm 7.6^{*}$ & $<0.001$ \\
\hline Male sex & 486 (73.5) & 348 (71.8) & $98(76.6)$ & $43(82.7)$ & 0.162 \\
\hline Smoking & $174(26.2)$ & $127(26.2)$ & $36(28.1)$ & $11(21.2)$ & 0.628 \\
\hline Diabetes mellitus & $94(14.1)$ & $54(11.1)$ & $27(21.1)^{*}$ & $13(25.0)^{*}$ & 0.001 \\
\hline Hypertension & $289(43.5)$ & $190(39.2)$ & $70(54.7)^{*}$ & $29(55.8)^{*}$ & 0.001 \\
\hline Lipid lowering medication & $38(5.7)$ & $21(4.3)$ & $12(9.4)^{*}$ & $5(9.6)$ & 0.041 \\
\hline Systolic blood pressure, mm Hg & $128.6 \pm 13.8$ & $127.8 \pm 13.7$ & $131.7 \pm 14.3^{*}$ & $129.2 \pm 11.7$ & 0.016 \\
\hline Diastolic blood pressure, $\mathrm{mm} \mathrm{Hg}$ & $77.8 \pm 9.9$ & $77.8 \pm 10.1$ & $78.7 \pm 9.1$ & $75.3 \pm 9.4$ & 0.115 \\
\hline Body mass index, $\mathrm{kg} / \mathrm{m}^{2}$ & $25.9 \pm 3.0$ & $25.7 \pm 2.9$ & $26.4 \pm 3.1$ & $26.4 \pm 2.7$ & 0.037 \\
\hline Waist circumference, $\mathrm{cm}$ & $89.1 \pm 8.1$ & $88.7 \pm 8.2$ & $90.2 \pm 7.7$ & $91.1 \pm 7.0$ & 0.031 \\
\hline Metabolic syndrome & 344 (51.7) & $231(47.6)$ & $80(62.5)^{*}$ & $33(63.5)^{*}$ & 0.002 \\
\hline Albumin, g/dL & $4.5 \pm 0.3$ & $4.55 \pm 0.26$ & $4.53 \pm 0.25$ & $4.54 \pm 0.28$ & 0.637 \\
\hline Bilirubin, mg/dL & $0.95 \pm 0.39$ & $0.97 \pm 0.39$ & $0.89 \pm 0.36$ & $0.97 \pm 0.43$ & 0.134 \\
\hline AST, IU/L & $23.2 \pm 9.9$ & $23.2 \pm 10.0$ & $23.2 \pm 10.1$ & $23.6 \pm 8.5$ & 0.97 \\
\hline ALT, IU/L & $31.2 \pm 22.2$ & $31.4 \pm 21.4$ & $31.7 \pm 25.8$ & $29.0 \pm 19.8$ & 0.737 \\
\hline GGT, IU/L & $40.7 \pm 38.0$ & $40.6 \pm 35.8$ & $40.1 \pm 37.6$ & $43.3 \pm 55.9$ & 0.869 \\
\hline Total cholesterol, mg/dL & $205.8 \pm 38.9$ & $205.2 \pm 37.7$ & $206.7 \pm 41.6$ & $209.0 \pm 43.6$ & 0.759 \\
\hline Triglyceride, mg/dL & $151.2 \pm 86.5$ & $148.8 \pm 91.0$ & $158.7 \pm 70.2$ & $155.1 \pm 79.4$ & 0.485 \\
\hline HDL cholesterol, mg/dL & $43.6 \pm 10.2$ & $43.8 \pm 10.1$ & $42.1 \pm 9.6$ & $45.0 \pm 11.7$ & 0.131 \\
\hline LDL cholesterol, mg/dL & $127.9 \pm 33.8$ & $128.0 \pm 32.6$ & $129.5 \pm 37.3$ & $123.6 \pm 36.4$ & 0.571 \\
\hline Fasting glucose, mg/dL & $102.7 \pm 25.0$ & $100.3 \pm 23.4$ & $108.3 \pm 28.0^{*}$ & $110.9 \pm 28.2$ & $<0.001$ \\
\hline Creatinine, mg/dL & $0.9 \pm 0.2$ & $0.87 \pm 0.17$ & $0.90 \pm 0.22$ & $0.92 \pm 0.20$ & 0.029 \\
\hline Platelet, $10^{3} / \mathrm{mm}^{3}$ & $250.0 \pm 53.2$ & $251.8 \pm 53.1$ & $248.5 \pm 54.0$ & $237.2 \pm 50.5$ & 0.162 \\
\hline Alcohol consumption, g/day & $7.6 \pm 8.4$ & $7.8 \pm 8.5$ & $6.2 \pm 7.8$ & $8.8 \pm 8.4$ & 0.084 \\
\hline NAFLD fibrosis score & $-2.266 \pm 1.120$ & $-2.428 \pm 1.078$ & $-1.955 \pm 1.105^{*}$ & $-1.516 \pm 1.094^{*, \dagger}$ & $<0.001$ \\
\hline FIB-4 score & $0.95 \pm 0.45$ & $0.90 \pm 0.38$ & $1.02 \pm 0.43^{*}$ & $1.26 \pm 0.83^{*, \dagger}$ & $<0.001$ \\
\hline Forn's index & $4.00 \pm 1.21$ & $3.86 \pm 1.16$ & $4.26 \pm 1.29^{*}$ & $4.64 \pm 1.18^{*}$ & $<0.001$ \\
\hline APRI & $0.26 \pm 0.14$ & $0.26 \pm 0.15$ & $0.26 \pm 0.14$ & $0.27 \pm 0.10$ & 0.791 \\
\hline
\end{tabular}

Data are presented as mean \pm SD or number (\%).

AST, aspartate transaminase; ALT, alanine transaminase; GGT, gamma-glutamyl transferase; HDL, high-density lipoprotein; LDL, low-density lipoprotein; NAFLD, nonalcoholic fatty liver disease; FIB-4, fibrosis-4; APRI, AST to platelet ratio index.

${ }^{*} \mathrm{p}<0.05$ vs $\mathrm{CACS}=0 ;{ }^{\dagger} \mathrm{p}<0.05$ vs $\mathrm{CACS}>0-100$.

jects (7.8\%) had a CACS over 100. As the CACS increased, age, BMI, waist circumference, serum fasting glucose level, and serum creatinine increased (all $\mathrm{p}<0.05$ ). The prevalence of diabetes, hypertension and metabolic syndrome increased (all $\mathrm{p}<0.01$ ). Moreover, noninvasive fibrosis markers, such as the NFS and the FIB-4 score and Forn's index, significantly increased as the CACS increased (all $\mathrm{p}<0.001$ ). CACS also had significant positive correlation with the NFS $(r=0.157, p<0.001)$, and the FIB4 score $(r=0.152, p<0.001)$, and Forn's index $(r=0.120, p=0.002)$ (Supplementary Table 1).

\section{Factors associated with CACS $>100$}

The results from multinomial regression logistic regression models that explored risk factor relationships with CACS $>0$ 100 and CACS $>100$ are displayed in Table 2 . At $>55$ years of age, the presence of hypertension and diabetes, elevated fasting plasma glucose, serum bilirubin level and triglyceride level were significant factors associated with CACS 0-100. At $>55$ years of age, the presence of hypertension and diabetes, BMI $\geq 25 \mathrm{~kg} /$ $\mathrm{m}^{2}$, elevated fasting plasma glucose and decreased eGFR were significant factors associated with CACS $>100$. Noninvasive fibrosis markers were significantly associated with CACS, except for the APRI for CACS 0-100. However, noninvasive steatosis markers were not associated with CACS, except for the FLI for CACS $>100$.

Univariate binary logistic regression analysis identified three 
Table 2. Factors Associated with Coronary Artery Calcium Score According to the Multinomial Logistic Regression Model in Nonalcoholic Fatty Liver Disease Patients

\begin{tabular}{|c|c|c|c|c|}
\hline \multirow{2}{*}{ Variable } & \multicolumn{2}{|c|}{ CACS $(>0-100)$} & \multicolumn{2}{|c|}{ CACS $(>100)$} \\
\hline & OR $(95 \%$ CI) & p-value & OR $(95 \% \mathrm{CI})$ & $\mathrm{p}$-value \\
\hline Age $>55 \mathrm{yr}$ & $2.069(1.390-3.080)$ & $<0.001$ & $5.784(3.078-10.867)$ & $<0.001$ \\
\hline Male sex & $1.286(0.817-2.025)$ & 0.278 & $1.881(0.893-3.963)$ & 0.097 \\
\hline Hypertension & $1.874(1.265-2.776)$ & 0.002 & $1.958(1.100-3.485)$ & 0.022 \\
\hline Diabetes & $2.134(1.281-3.554)$ & 0.004 & $2.660(1.336-5.296)$ & 0.005 \\
\hline Smoking & $1.103(0.714-1.705)$ & 0.659 & $0.756(0.377-1.516)$ & 0.756 \\
\hline $\mathrm{BMI} \geq 25 \mathrm{~kg} / \mathrm{m}^{2}$ & $1.273(0.853-1.899)$ & 0.238 & $1.884(1.007-3.523)$ & 0.048 \\
\hline $\mathrm{WC} \geq 90 \mathrm{~cm}$ (male) or $\geq 80 \mathrm{~cm}$ (female) & $1.274(0.856-1.896)$ & 0.233 & $1.681(0.917-3.083)$ & 0.093 \\
\hline Albumin $<4.5 \mathrm{~g} / \mathrm{dL}$ & $0.874(0.591-1.292)$ & 0.500 & $1.231(0.694-2.184)$ & 0.478 \\
\hline Bilirubin $>1.0 \mathrm{mg} / \mathrm{dL}$ & $0.599(0.382-0.940)$ & 0.026 & $0.951(0.517-1.748)$ & 0.871 \\
\hline $\mathrm{eGFR}<90 \mathrm{~mL} / \mathrm{min} / 1.73 \mathrm{~m}^{2}$ & $1.395(0.934-2.085)$ & 0.104 & $2.699(1.382-5.272)$ & 0.004 \\
\hline $\mathrm{LDL} \geq 130 \mathrm{mg} / \mathrm{dL}$ & $1.030(0.697-1.522)$ & 0.882 & $0.638(0.350-1.160)$ & 0.638 \\
\hline $\mathrm{TG} \geq 150 \mathrm{mg} / \mathrm{dL}$ & $1.653(1.116-2.449)$ & 0.012 & $1.453(0.819-2.579)$ & 0.202 \\
\hline $\mathrm{HDL}<40 \mathrm{mg} / \mathrm{dL}$ (male) or $<50 \mathrm{mg} / \mathrm{dL}$ (female) & $1.282(0.867-1.896)$ & 0.213 & $1.029(0.581-1.824)$ & 0.921 \\
\hline \multicolumn{5}{|l|}{ Noninvasive steatosis marker } \\
\hline Hepatic steatosis index & $0.999(0.951-1.049)$ & 0.999 & $0.939(0.872-1.011)$ & 0.094 \\
\hline Fatty liver index & $1.009(0.998-1.020)$ & 0.100 & $1.019(1.004-1.035)$ & 0.015 \\
\hline \multicolumn{5}{|l|}{ Noninvasive fibrosis marker } \\
\hline NAFLD fibrosis score $(>-2.745)$ & $1.675(1.094-2.564)$ & 0.018 & $7.863(2.790-22.161)$ & $<0.001$ \\
\hline FIB-4 (>0.85) & $1.788(1.202-2.660)$ & 0.004 & $5.475(2.612-11.479)$ & $<0.001$ \\
\hline Forn’s index (>3.8) & $1.711(1.138-2.572)$ & 0.01 & $3.635(1.783-7.410)$ & $<0.001$ \\
\hline APRI (>0.219) & $1.219(0.823-1.803)$ & 0.323 & $2.843(1.481-5.460)$ & 0.002 \\
\hline
\end{tabular}

Reference category: $\mathrm{CACS}=0$

CACS, coronary artery calcium score; OR, odds ratio; CI, confidential interval; BMI, body mass index; WC, waist circumference; eGFR, estimated glomerular filtration rate; LDL, low-density lipoprotein; TG, triglyceride; HDL, high-density lipoprotein; NAFLD, nonalcoholic fatty liver disease; FIB-4, fibrosis-4; APRI, aspartate aminotransferase to platelet ratio index.

significant factors and two factors with marginal significance associated with CACS $>100$ (Table 3 ). At $>55$ years of age, the presence of diabetes mellitus and eGFR (all $\mathrm{p}<0.05$ ) were statistically significant factors, and the presence of hypertension and $\mathrm{BMI} \geq 25 \mathrm{~kg} / \mathrm{m}^{2}$ were factors with marginal significance associated with CACS $>100(\mathrm{p}=0.065$ and $\mathrm{p}=0.065)$. Multivariate binary logistic regression analysis that included these factors revealed that older age, elevated BMI and decreased eGFR were significant factors for predicting CACS $>100(\mathrm{p}<0.001, \mathrm{p}=0.016$, and $\mathrm{p}=0.028$, respectively) (Table 3 ).

To assess the association between noninvasive steatosis and fibrosis markers and CACS $>100$, we performed multivariate analysis adjusted for potential confounders (Table 4). In model 1 , which was adjusted for age and gender, the NFS and the FIB4 score and APRI were significant predictive factors for CACS $>100$. In model 2, which was adjusted for age, gender, BMI and eGFR, the NFS and the FIB-4 score and APRI were also significant predictive factors for CACS $>100$. Unlike noninvasive fibrosis markers, noninvasive steatosis markers were not significant factors for CACS >100 except FLI in model 1.

\section{Performance of noninvasive fibrosis markers in predict- ing coronary artery calcification}

The performance of the noninvasive fibrosis markers in predicting CACS $>100$ in the subjects with NAFLD was evaluated by ROC curves (Fig. 2). Table 4 shows the AUROC for noninvasive fibrosis markers in predicting CACS $>100$. The AUROCs for the NFS and the FIB-4 score, Forn's index and APRI were $0.689,0.683,0.659$, and 0.595 , respectively. There were no significant differences among the AUROCs, except between the NFS and APRI ( $p=0.028)$ and between the FIB-4 score and APRI $(p=0.008)$. The best performance levels for the NFS and the FIB4 score, Forn's index, and APRIs were observed at cutoff values of $-2.745,0.85,3.8$ and 0.219 , respectively. These cutoff values exhibited sensitivity and specificity values of 92.3\% and 37.2\% for the NFS, $82.7 \%$ and 50.4\% for the FIB-4 score, 80.8\% and $43.7 \%$ for Forn's index, and $75.0 \%$ and $47.6 \%$ for the APRI, respectively.

Because the performance of original noninvasive fibrosis markers was less predictive, new prognostic models, integrating 
Table 3. Factors Associated with a Coronary Calcium Score $>100$ in Subjects with Nonalcoholic Fatty Liver Disease

\begin{tabular}{|c|c|c|c|c|}
\hline \multirow{2}{*}{ Variable } & \multicolumn{2}{|c|}{ Univariate } & \multicolumn{2}{|c|}{ Multivariate } \\
\hline & OR $(95 \% \mathrm{CI})$ & p-value & OR (95\% CI) & p-value \\
\hline Age $>55$ yr & $4.909(2.633-9.153)$ & $<0.001$ & $4.809(2.556-9.049)$ & $<0.001$ \\
\hline Male sex & $1.789(0.853-3.750)$ & 0.123 & & \\
\hline Hypertension & $1.712(0.968-3.028)$ & 0.065 & & \\
\hline Diabetes & $2.189(1.120-4.278)$ & 0.022 & & \\
\hline Smoking & $0.741(0.372-1.476)$ & 0.393 & & \\
\hline $\mathrm{BMI} \geq 25 \mathrm{~kg} / \mathrm{m}^{2}$ & $1.055(0.962-1.157)$ & 0.065 & $2.072(1.093-3.928)$ & 0.026 \\
\hline $\mathrm{WC} \geq 90 \mathrm{~cm}$ (male) or $\geq 80 \mathrm{~cm}$ (female) & $1.599(0.877-2.917)$ & 0.126 & & \\
\hline Albumin, g/dL & $0.914(0.304-2.748)$ & 0.873 & & \\
\hline Bilirubin, mg/dL & $1.127(0.555-2.289)$ & 0.74 & & \\
\hline $\mathrm{eGFR}<90 \mathrm{~mL} / \mathrm{min} / 1.73 \mathrm{~m}^{2}$ & $2.521(1.297-4.901)$ & 0.006 & $2.149(1.088-4.245)$ & 0.028 \\
\hline Cholesterol, mg/dL & 1.002 (0.995-1.010) & 0.531 & & \\
\hline $\mathrm{LDL}, \mathrm{mg} / \mathrm{dL}$ & $0.996(0.987-1.004)$ & 0.339 & & \\
\hline $\mathrm{TG} \geq 150 \mathrm{mg} / \mathrm{dL}$ & $1.308(0.741-2.308)$ & 0.354 & & \\
\hline $\mathrm{HDL}<40 \mathrm{mg} / \mathrm{dL}$ (male) or $<50 \mathrm{mg} / \mathrm{dL}$ (female) & $0.977(0.555-1.722)$ & 0.937 & & \\
\hline \multicolumn{5}{|l|}{ Noninvasive steatosis marker } \\
\hline Hepatic steatosis index & $0.991(0.939-1.047)$ & 0.750 & & \\
\hline Fatty liver index & $1.008(0.997-1.020)$ & 0.155 & & \\
\hline \multicolumn{5}{|l|}{ Noninvasive fibrosis marker } \\
\hline NAFLD fibrosis score & $1.943(1.488-2.537)$ & $<0.001$ & & \\
\hline FIB-4 & $3.018(1.759-5.177)$ & $<0.001$ & & \\
\hline Forn's index & $1.605(1.269-2.030)$ & $<0.001$ & & \\
\hline APRI & $1.804(0.321-10.124)$ & 0.503 & & \\
\hline
\end{tabular}

OR, odds ratio; CI, confidential interval; BMI, body mass index; WC, waist circumference; eGFR, estimated glomerular filtration rate; LDL, lowdensity lipoprotein; TG, triglyceride; HDL, high-density lipoprotein; NAFLD, nonalcoholic fatty liver disease; FIB-4, fibrosis-4; APRI, aspartate aminotransferase to platelet ratio index.

Table 4. Associations between Noninvasive Serum Fibrosis Markers and a Coronary Calcium Score $>100$ in Subjects with Nonalcoholic Fatty Liver Disease

\begin{tabular}{|c|c|c|c|c|}
\hline & \multicolumn{2}{|c|}{ Model 1} & \multicolumn{2}{|c|}{ Model 2} \\
\hline & OR $(95 \%$ CI $)$ & p-value & OR $(95 \% \mathrm{CI})$ & p-value \\
\hline NAFLD fibrosis score $(>-2.745)$ & $4.375(1.511-12.663)$ & 0.006 & $3.91(1.339-11.416)$ & 0.013 \\
\hline FIB-4 score $(>0.85)$ & $2.791(1.248-6.243)$ & 0.012 & $2.573(1.147-5.769)$ & 0.022 \\
\hline Forn’s index (>3.8) & $1.539(0.698-3.396)$ & 0.285 & $1.536(0.698-3.383)$ & 0.286 \\
\hline APRI (>0.219) & $2.365(1.212-4.615)$ & 0.012 & $2.151(1.093-4.231)$ & 0.027 \\
\hline Hepatic steatosis index & 1.017 (0.959-1.077) & 0.578 & $0.99(0.920-1.065)$ & 0.783 \\
\hline Fatty liver index & $1.013(1.000-1.027)$ & 0.049 & $1.009(0.994-1.026)$ & 0.243 \\
\hline
\end{tabular}

Model 1: adjusted for age ( $\geq 55$ years of age) and gender. Model 2 : adjusted for age ( $\geq 55$ years of age), gender, $\mathrm{BMI}\left(\geq 25 \mathrm{~kg} / \mathrm{m}^{2}\right)$, and eGFR $<90 \mathrm{~mL} /$ $\min / 1.73 \mathrm{~m}^{2}$.

OR, odds ratio; CI, confidential interval; NAFLD, nonalcoholic fatty liver disease; FIB-4, fibrosis-4; APRI, aspartate transaminase to platelet ratio index; BMI, body mass index; eGFR, estimated glomerular filtration rate.

the noninvasive fibrosis markers and the risk factors for $\mathrm{CAD}$ (older age, gender, elevated BMI and decreased eGFR), were developed based on the results obtained from the multivariable logistic regression model (Supplementary Table 2).

The AUROCs for the NFS model, the FIB-4 score model, Forn's index model and APRI model were increased to 0.797, 0.785, 0.775 , and 0.782 , respectively. The best performance levels for the NFS model, the FIB-4 score model, Forn's index model, and APRI model were observed at cutoff values of $-2.459,-2.442$, -2.441 , and -2.231 , respectively. These cutoff values exhibited 

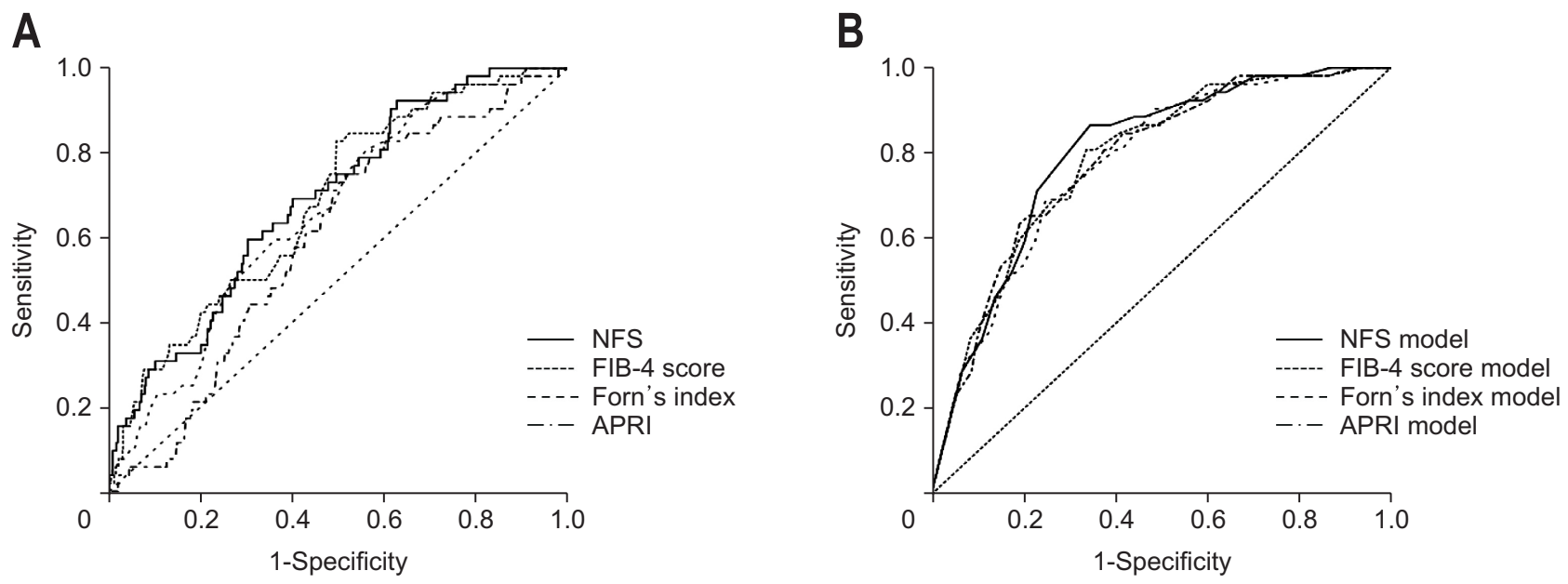

Fig. 2. Receiver operating characteristics curve for noninvasive serum fibrosis markers predicting a coronary calcium score $>100$ among subjects with nonalcoholic fatty liver disease (NAFLD). (A) Original noninvasive fibrosis markers. (B) New models integrating noninvasive markers and risk factors for coronary artery disease.

NFS, NAFLD fibrosis score; FIB-4, fibrosis-4; APRI, aspartate aminotransferase to platelet ratio index.

Table 5. Performance of Noninvasive Fibrosis Markers in Predicting a Coronary Calcium Score >100 Using the Optimal Cutoff Point

\begin{tabular}{|c|c|c|c|c|c|c|}
\hline Variable & AUROC & Cutoff value & Sensitivity, \% & Specificity, \% & PPV, $\%$ & NPV, $\%$ \\
\hline \multicolumn{7}{|l|}{ Original score } \\
\hline NAFLD fibrosis score & 0.689 & -2.745 & 92.3 & 37.2 & 11.1 & 98.3 \\
\hline FIB-4 & 0.683 & 0.85 & 82.7 & 50.4 & 12.4 & 97.2 \\
\hline Forn's index & 0.659 & 3.8 & 80.8 & 43.7 & 10.9 & 96.4 \\
\hline APRI & 0.595 & 0.219 & 75.0 & 47.6 & 10.8 & 95.7 \\
\hline \multicolumn{7}{|l|}{ New model } \\
\hline NAFLD fibrosis score model & 0.797 & -2.459 & 86.5 & 65.4 & 17.5 & 98.3 \\
\hline FIB-4 score model & 0.785 & -2.442 & 80.8 & 66.2 & 16.9 & 97.6 \\
\hline Forn's index model & 0.775 & -2.441 & 69.2 & 75.0 & 19.0 & 96.6 \\
\hline APRI model & 0.782 & -2.231 & 65.4 & 78.8 & 20.7 & 96.4 \\
\hline
\end{tabular}

AUROC, area under receiver operating characteristic curve; PPV, positive predictive value; NPV, negative predictive value; NAFLD, nonalcoholic fatty liver disease; FIB-4, fibrosis-4; APRI, aspartate aminotransferase to platelet ratio index.

sensitivity and specificity values of $86.5 \%$ and $65.4 \%$ for the NFS model, $80.8 \%$ and $66.2 \%$ for the FIB- 4 score model, $69.2 \%$ and $75.0 \%$ for Forn's index model, and $65.4 \%$ and $78.8 \%$ for the APRI model, respectively (Table 5).

Because age was the most significant predictive factor, we analyzed the predictive power of noninvasive fibrosis markers for CACS >100 (Table 6). The NFS and the FIB-4 score, and APRI were significant factors in the older age group, but not in the younger age group.

\section{DISCUSSION}

In this study, we found that noninvasive fibrosis markers, such as the NFS and the FIB-4 score and Forn's index scores, significantly increased as the CACS increased. Noninvasive fibrosis markers were independently associated with CACS $>100$. Moreover, new scores integrating noninvasive fibrosis markers
Table 6. Associations between Noninvasive Fibrosis Markers and CACS $>100$ Stratified by Age

\begin{tabular}{lcc}
\hline \multicolumn{1}{c}{ Variable } & OR $(95 \% \mathrm{CI})$ & $\mathrm{p}$-value \\
\hline Age $<55$ yr & & \\
NAFLD fibrosis score & $3.521(0.979-12.663)$ & 0.054 \\
FIB-4 & $2.141(0.761-6.024)$ & 0.149 \\
Forn's index & $1.364(0.486-3.832)$ & 0.556 \\
APRI & $2.518(0.789-8.037)$ & 0.119 \\
Age $\geq 55$ yr & & \\
NAFLD fibrosis score & $7.929(1.053-59.686)$ & 0.044 \\
FIB-4 & $4.645(1.074-20.083)$ & 0.04 \\
Forn's index & $3.279(0.963-11.164)$ & 0.057 \\
APRI & $2.794(1.256-6.216)$ & 0.012 \\
\hline
\end{tabular}

CACS, coronary artery calcium score; OR, odds ratio; CI, confidence interval; NAFLD, nonalcoholic fatty liver disease; FIB-4, fibrosis-4; APRI, aspartate aminotransferase to platelet ratio index. 
and risk factors for $\mathrm{CAD}$ showed good discriminatory power in predicting CACS $>100$.

Previous cross-sectional studies and meta-analyses showed that NAFLD was associated with subclinical atherosclerosis, independent of traditional CVD risk factors. ${ }^{23,24}$ NAFLD is also associated with the progression of subclinical atherosclerosis markers, such as CIMT and CAC. ${ }^{25,26}$ The CACS measured by cardiac CT represents the atherosclerotic burden of coronary artery. In addition, the CACS is associated with future cardiovascular events in several studies. ${ }^{24,27,28}$ In our study, subjects with a high CACS tended to have more metabolic syndrome features, such as hypertension, diabetes, high BMI, and high waist circumference (Table 1), and old age, elevated BMI and decreased eGFR were significantly associated with a high CACS after adjusting for these metabolic features (Table 3). These results are expected, considering that variables related to metabolic syndrome, renal disease and age are well-established risk factors for CVD. ${ }^{29,30}$ In addition to these well-established risk factors, noninvasive fibrosis markers were also significantly associated with a high CACS in our study. Our results suggest that the risk of CAD increases as hepatic fibrosis progresses in subjects with NAFLD. This finding is consistent with data from You et al. ${ }^{31}$ who showed that the liver stiffness value measured by transient elastography (TE) is associated with a higher CACS in subjects with NAFLD. Our study also showed that noninvasive fibrosis markers combined with risk factors for $\mathrm{CAD}$ showed good performance in predicting a high CACS (>100), which is associated with future CVD (Fig. 2). Therefore, noninvasive fibrosis markers could be useful tools for identifying subjects with a high risk for CVD development.

Previous studies have reported that CVD is a major cause of death among subjects with NAFLD, and the presence and stage of hepatic fibrosis are the main determinants of mortality from CVD or liver-related disease. ${ }^{3,5,10,32}$ Although liver biopsy remains the gold standard for assessing the fibrosis stage in patients with NAFLD, thus far, it is impractical for widespread use given the high prevalence of NAFLD. Noninvasive methods to assess hepatic fibrosis include imaging techniques and serum biomarkers. The most validated imaging modality in patients with NAFLD is TE, which showed high performance for advanced fibrosis (stage 3 or 4) (sensitivity 85\%-92\%, specificity 82\%-92\%). ${ }^{12,33,34}$ However, TE could be limited in obese patients due to measurement failure or unreliable results, although the use of the XL probe improved these limitations. ${ }^{35-37}$ In addition, there were conflicting data regarding the effect of hepatic steatosis on the liver stiffness value of TE. ${ }^{38,39}$ Magnetic resonance elastography (MRE) analyzes almost the entire liver and has good applicability in patients with obesity. Some studies have reported that MRE is more accurate than TE for the diagnosis of advanced fibrosis. ${ }^{40-42}$ However, MRE is too costly and time-consuming and therefore is not suitable for routine screening of NAFLD patients in clinical practice. On the other hand, serum biomarkers have the advantages of low cost, high applicability (>95\%), and widespread availability. ${ }^{12}$ In addition, serum biomarkers, such as the NFS and FIB-4 score, have been well-validated in subjects with NAFLD, which shows that these markers have good performance in diagnosing advanced fibrosis. ${ }^{43}$ Therefore, serum biomarkers are an adequate modality that can be easily used to identify patients with advanced fibrosis in routine clinical practice.

NAFLD is associated with increased risk of chronic kidney disease (CKD), and metabolic status in NAFLD patients impacts on CKD development. ${ }^{44,45}$ Coronary artery calcification is highly prevalent and more severe in patients with $\mathrm{CKD} .{ }^{46}$ In addition, CKD is strongly associated with development of CVD. ${ }^{47}$ In CKD patients, coronary artery calcification is associated with systemic inflammation. ${ }^{48}$ In the same manner, CAD in NAFLD patients is also associated with systemic inflammation. ${ }^{49}$ Therefore, it is natural that decreased eGFR was a significant factor for predicting CACS $>100$, consistent with a previous study. ${ }^{31}$

Our study has some limitations. First, the cross-sectional design makes it difficult to determine the causal relationships between noninvasive fibrosis markers and coronary artery calcifications. Second, the absence of a liver biopsy as a reference standard for the diagnosis of fatty liver and degree of liver fibrosis could be a limitation. We diagnosed NAFLD by ultrasonography, which has difficulty in identifying fatty infiltration below 30\%, and the intra- and inter-observer variability can affect the diagnosis. ${ }^{50}$ However, ultrasonography has the advantages of safety, relatively low cost, repeatability, high sensitivity and specificity. ${ }^{51}$ Given these strengths, ultrasonography could be a good imaging technique for diagnosing NAFLD in the general population. In addition, although there could be discordance between noninvasive markers and liver biopsy, NFS and FIB-4 score are more adequate than liver biopsy in clinical practice due to the low prevalence of significant fibrosis, as shown in our study. Third, selection bias could have been involved because subjects with high CVD risk are more likely to have a coronary CT scan in a health check-up. Indeed, included subjects were older and had higher noninvasive fibrosis markers (Supplementary Table 3). However, noninvasive fibrosis markers were independently associated with high CACS after adjusting for other risk factors. Fourth, while original noninvasive fibrosis markers and new models integrating noninvasive fibrosis markers and risk factors for $\mathrm{CAD}$ showed high sensitivity and NPV for predicting high CACS, their specificity and PPV were too low. This result might be caused by low prevalence of high CACS (7.8\%). Fifth, because we investigated some data using a self-reporting questionnaire, we should consider the possibility of a response bias. In addition, data investigation by questionnaire makes it difficult to identify subjects who are taking drugs that could affect hepatic fibrosis and coronary calcification, such as an angiotensin II receptor antagonist or statin. ${ }^{52,53}$ Therefore, prospective studies are necessary to investigate the 
causal relationship and eliminate the effect of confounding factors between hepatic fibrosis and coronary subclinical atherosclerosis in subjects with NAFLD. In addition, the cutoff values of noninvasive fibrosis markers and new models to discriminate patients with risk of CAD should be validated in further studies.

In conclusion, high noninvasive fibrosis markers are significantly associated with a high CACS in subjects with NAFLD. Moreover, new models integrating noninvasive markers and the risk factors for $\mathrm{CAD}$ showed good discriminatory power for predicting CACS >100. Therefore, noninvasive fibrosis markers and risk factors for CAD may be useful tools for identifying NAFLD subjects with a high risk of future CVD development.

\section{CONFLICTS OF INTEREST}

No potential conflict of interest relevant to this article was reported.

\section{AUTHOR CONTRIBUTIONS}

Study concept and design: D.S.S. Acquisition of data: S.G.K., S.W.S. Analysis and interpretation of data: D.S.S. U.I.C. Drafting of the manuscript: D.S.S. Critical revision of the manuscript: J.M.Y. Statistical analysis. D.S.S. Approval of final manuscript: all authors.

\section{ORCID}

Do Seon Song https://orcid.org/0000-0002-8278-4878

U Im Chang https://orcid.org/0000-0002-6937-2895

Sung-Goo Kang https://orcid.org/0000-0003-4627-291X

Sang-Wook Song https://orcid.org/0000-0001-9087-5019

Jin Mo Yang https://orcid.org/0000-0002-8339-4716

\section{REFERENCES}

1. Chalasani N, Younossi Z, Lavine JE, et al. The diagnosis and management of non-alcoholic fatty liver disease: practice guideline by the American Gastroenterological Association, American Association for the Study of Liver Diseases, and American College of Gastroenterology. Gastroenterology 2012;142:1592-1609.

2. Younossi ZM, Koenig AB, Abdelatif D, Fazel Y, Henry L, Wymer M. Global epidemiology of nonalcoholic fatty liver disease-metaanalytic assessment of prevalence, incidence, and outcomes. Hepatology 2016;64:73-84.

3. Ekstedt M, Hagström H, Nasr P, et al. Fibrosis stage is the strongest predictor for disease-specific mortality in NAFLD after up to 33 years of follow-up. Hepatology 2015;61:1547-1554.

4. Angulo P, Kleiner DE, Dam-Larsen S, et al. Liver fibrosis, but no other histologic features, is associated with long-term outcomes of patients with nonalcoholic fatty liver disease. Gastroenterology 2015;149:389-397.
5. Adams LA, Lymp JF, St Sauver J, et al. The natural history of nonalcoholic fatty liver disease: a population-based cohort study. Gastroenterology 2005;129:113-121.

6. Wong RJ, Aguilar M, Cheung R, et al. Nonalcoholic steatohepatitis is the second leading etiology of liver disease among adults awaiting liver transplantation in the United States. Gastroenterology 2015;148:547-555.

7. Kim D, Choi SY, Park EH, et al. Nonalcoholic fatty liver disease is associated with coronary artery calcification. Hepatology 2012;56:605-613.

8. Jaruvongvanich V, Wirunsawanya K, Sanguankeo A, Upala S. Nonalcoholic fatty liver disease is associated with coronary artery calcification: a systematic review and meta-analysis. Dig Liver Dis 2016;48:1410-1417.

9. Targher G, Bertolini L, Padovani R, et al. Relations between carotid artery wall thickness and liver histology in subjects with nonalcoholic fatty liver disease. Diabetes Care 2006;29:1325-1330.

10. Ong JP, Pitts A, Younossi ZM. Increased overall mortality and liver-related mortality in non-alcoholic fatty liver disease. J Hepatol 2008;49:608-612.

11. Patel K, Bedossa P, Castera L. Diagnosis of liver fibrosis: present and future. Semin Liver Dis 2015;35:166-183.

12. European Association for Study of Liver; Asociacion Latinoamericana para el Estudio del Higado. EASL-ALEH Clinical Practice Guidelines: non-invasive tests for evaluation of liver disease severity and prognosis. J Hepatol 2015;63:237-264.

13. Bedossa P, Patel K. Biopsy and noninvasive methods to assess progression of nonalcoholic fatty liver disease. Gastroenterology 2016;150:1811-1822.

14. Agatston AS, Janowitz WR, Hildner FJ, et al. Quantification of coronary artery calcium using ultrafast computed tomography. J Am Coll Cardiol 1990;15:827-832.

15. Hajar R. Risk factors for coronary artery disease: historical perspectives. Heart Views 2017;18:109-114.

16. Levey AS, Bosch JP, Lewis JB, Greene T, Rogers N, Roth D. A more accurate method to estimate glomerular filtration rate from serum creatinine: a new prediction equation. Modification of Diet in Renal Disease Study Group. Ann Intern Med 1999;130:461470.

17. Lee JH, Kim D, Kim HJ, et al. Hepatic steatosis index: a simple screening tool reflecting nonalcoholic fatty liver disease. Dig Liver Dis 2010;42:503-508.

18. Bedogni G, Bellentani S, Miglioli L, et al. The Fatty Liver Index: a simple and accurate predictor of hepatic steatosis in the general population. BMC Gastroenterol 2006;6:33.

19. Angulo P, Hui JM, Marchesini G, et al. The NAFLD fibrosis score: a noninvasive system that identifies liver fibrosis in patients with NAFLD. Hepatology 2007;45:846-854.

20. Vallet-Pichard A, Mallet V, Nalpas B, et al. FIB-4: an inexpensive and accurate marker of fibrosis in HCV infection. comparison with liver biopsy and fibrotest. Hepatology 2007;46:32-36.

21. Forn'ss X, Ampurdanès S, Llovet JM, et al. Identification of chron- 
ic hepatitis C patients without hepatic fibrosis by a simple predictive model. Hepatology 2002;36(4 Pt 1):986-992.

22. Wai CT, Greenson JK, Fontana RJ, et al. A simple noninvasive index can predict both significant fibrosis and cirrhosis in patients with chronic hepatitis C. Hepatology 2003;38:518-526.

23. Kim NH, Park J, Kim SH, et al. Non-alcoholic fatty liver disease, metabolic syndrome and subclinical cardiovascular changes in the general population. Heart 2014;100:938-943.

24. Detrano R, Guerci AD, Carr JJ, et al. Coronary calcium as a predictor of coronary events in four racial or ethnic groups. N Engl J Med 2008;358:1336-1345.

25. Sinn DH, Kang D, Chang Y, et al. Non-alcoholic fatty liver disease and progression of coronary artery calcium score: a retrospective cohort study. Gut 2017;66:323-329.

26. Sinn DH, Cho SJ, Gu S, et al. Persistent nonalcoholic fatty liver disease increases risk for carotid atherosclerosis. Gastroenterology 2016;151:481-488

27. Polonsky TS, McClelland RL, Jorgensen NW, et al. Coronary artery calcium score and risk classification for coronary heart disease prediction. JAMA 2010;303:1610-1616.

28. Greenland P, LaBree L, Azen SP, Doherty TM, Detrano RC. Coronary artery calcium score combined with Framingham score for risk prediction in asymptomatic individuals. JAMA 2004;291:210215.

29. Wilson PW, D’Agostino RB, Levy D, Belanger AM, Silbershatz H, Kannel WB. Prediction of coronary heart disease using risk factor categories. Circulation 1998;97:1837-1847.

30. Sarnak MJ, Levey AS, Schoolwerth AC, et al. Kidney disease as a risk factor for development of cardiovascular disease: a statement from the American Heart Association Councils on Kidney in Cardiovascular Disease, High Blood Pressure Research, Clinical Cardiology, and Epidemiology and Prevention. Hypertension 2003;42:1050-1065.

31. You SC, Kim KJ, Kim SU, et al. Hepatic fibrosis assessed using transient elastography independently associated with coronary artery calcification. J Gastroenterol Hepatol 2015;30:1536-1542.

32. Kim D, Kim WR, Kim HJ, Therneau TM. Association between noninvasive fibrosis markers and mortality among adults with nonalcoholic fatty liver disease in the United States. Hepatology 2013;57:1357-1365.

33. Hannah WN Jr, Harrison SA. Noninvasive imaging methods to determine severity of nonalcoholic fatty liver disease and nonalcoholic steatohepatitis. Hepatology 2016;64:2234-2243.

34. Kwok R, Tse YK, Wong GL, et al. Systematic review with metaanalysis: non-invasive assessment of non-alcoholic fatty liver disease. The role of transient elastography and plasma cytokeratin-18 fragments. Aliment Pharmacol Ther 2014;39:254-269.

35. Castéra L, Foucher J, Bernard PH, et al. Pitfalls of liver stiffness measurement: a 5-year prospective study of 13,369 examinations. Hepatology 2010;51:828-835.

36. Wong GL, Wong VW, Chim AM, et al. Factors associated with unreliable liver stiffness measurement and its failure with transient elastography in the Chinese population. J Gastroenterol Hepatol 2011;26:300-305.

37. Myers RP, Pomier-Layrargues G, Kirsch R, et al. Feasibility and diagnostic performance of the FibroScan XL probe for liver stiffness measurement in overweight and obese patients. Hepatology 2012;55:199-208.

38. Wong VW, Vergniol J, Wong GL, et al. Diagnosis of fibrosis and cirrhosis using liver stiffness measurement in nonalcoholic fatty liver disease. Hepatology 2010;51:454-462.

39. Gaia S, Carenzi S, Barilli AL, et al. Reliability of transient elastography for the detection of fibrosis in non-alcoholic fatty liver disease and chronic viral hepatitis. J Hepatol 2011;54:64-71.

40. Imajo K, Kessoku T, Honda Y, et al. Magnetic resonance imaging more accurately classifies steatosis and fibrosis in patients with nonalcoholic fatty liver disease than transient elastography. Gastroenterology 2016;150:626-637.

41. Huwart L, Sempoux C, Vicaut E, et al. Magnetic resonance elastography for the noninvasive staging of liver fibrosis. Gastroenterology 2008;135:32-40.

42. Ichikawa S, Motosugi U, Morisaka H, et al. Comparison of the diagnostic accuracies of magnetic resonance elastography and transient elastography for hepatic fibrosis. Magn Reson Imaging 2015;33:26-30

43. Castera L, Vilgrain V, Angulo P. Noninvasive evaluation of NAFLD. Nat Rev Gastroenterol Hepatol 2013;10:666-675.

44. Sinn DH, Kang D, Jang HR, et al. Development of chronic kidney disease in patients with non-alcoholic fatty liver disease: a cohort study. J Hepatol 2017;67:1274-1280.

45. Ampuero J, Aller R, Gallego-Durán R, et al. The effects of metabolic status on non-alcoholic fatty liver disease-related outcomes, beyond the presence of obesity. Aliment Pharmacol Ther 2018;48:1260-1270.

46. Kestenbaum BR, Adeney KL, de Boer IH, Ix JH, Shlipak MG, Siscovick DS. Incidence and progression of coronary calcification in chronic kidney disease: the Multi-Ethnic Study of Atherosclerosis. Kidney Int 2009;76:991-998.

47. Tonelli M, Muntner P, Lloyd A, et al. Risk of coronary events in people with chronic kidney disease compared with those with diabetes: a population-level cohort study. Lancet 2012;380:807-814.

48. Hwang IC, Park HE, Kim HL, et al. Systemic Inflammation Is Associated With Coronary Artery Calcification and All-Cause Mortality in Chronic Kidney Disease. Circ J 2016;80:1644-1652.

49. Byrne CD, Targher G. NAFLD: a multisystem disease. J Hepatol 2015;62(1 Suppl):S47-S64.

50. Oh H, Jun DW, Saeed WK, Nguyen MH. Non-alcoholic fatty liver diseases: update on the challenge of diagnosis and treatment. Clin Mol Hepatol 2016;22:327-335.

51. Saadeh S, Younossi ZM, Remer EM, et al. The utility of radiological imaging in nonalcoholic fatty liver disease. Gastroenterology 2002;123:745-750.

52. Zhu Q, Li N, Li F, et al. Therapeutic effect of renin angiotensin system inhibitors on liver fibrosis. J Renin Angiotensin Aldosterone 
668 Gut and Liver, Vol. 13, No. 6, November 2019

Syst 2016;17:1470320316628717.

53. Kamal S, Khan MA, Seth A, et al. Beneficial effects of statins on the rates of hepatic fibrosis, hepatic decompensation, and mortal- ity in chronic liver disease: a systematic review and meta-analysis. Am J Gastroenterol 2017;112:1495-1505. 